\title{
Nonresonant inelastic light scattering in the Hubbard model
}

\author{
J.K. Freericks*, T.P. Devereaux ${ }^{\dagger}$, R. Bulla ${ }^{\ddagger}$, and Th. Pruschke ${ }^{\ddagger}$ \\ * Department of Physics, Georgetown University, Washington, DC 20057, U.S.A. \\ ${ }^{\dagger}$ Department of Physics, University of Waterloo, ON N2L 3 G1 Canada \\ ${ }^{\ddagger}$ Theoretische Physik III, Elektronische Korrelationen und Magnetismus, Institut für Physik, Universität Augsburg, D-86135 \\ Augsburg, Germany
}

(July 3, 2018)

\begin{abstract}
Inelastic light scattering from electrons is a symmetry-selective probe of the charge dynamics within correlated materials. Many measurements have been made on correlated insulators, and recent exact solutions in large dimensions explain a number of anomalous features found in experiments. Here we focus on the correlated metal, as described by the Hubbard model away from half filling. We can determine the $B_{1 \mathrm{~g}}$ Raman response and the inelastic X-ray scattering along the Brillouin zone diagonal exactly in the large dimensional limit. We find a number of interesting features in the light scattering response which should be able to be seen in correlated metals such as the heavy fermions.
\end{abstract}

Principle: 78.30.-j; 71.30.+h; 74.72.-h

\section{INTRODUCTION}

Inelastic light scattering, and more specifically, electronic Raman and inelastic X-ray scattering, are useful probes of the two-particle charge excitations within a correlated metal. Experiments are extremely difficult to carry out, and so far, the only correlated metals that have been extensively studied are the high-temperature superconductors ${ }^{1-3}$. An experimental challenge is to have a large enough energy window for the inelastic light scattering to be able to see the higher-energy chargetransfer excitations as well as low-energy particle-hole excitations. One class of promising materials that could yield interesting Raman scattering results is the heavyfermion compounds that have sharply renormalized fermi temperatures, which compress the charge transfer excitations to a lower energy range (and thereby require a smaller energy window for the scattering experiments).

On the theoretical side, much progress has been made in examining the inelastic light scattering of correlated materials. The first breakthrough occurred over a decade ago when Shastry and Shraiman (SS) ${ }^{4}$ suggested a simplification to the theory of nonresonant Raman scattering where in certain cases the nonresonant Raman response may be equal to the optical conductivity multiplied by the frequency. Their conjecture was recently proved ${ }^{5}$ on a hypercubic lattice in $d \rightarrow \infty$ for $B_{1 g}$ polarization orientations. Subsequent work on the Hubbard model ${ }^{6}$ and on the Falicov-Kimball model ${ }^{7,5}$ has shown that Raman scattering is model independent in the insulating phase, and possesses all of the anomalous features seen in experiment on correlated insulators.

In this contribution, we focus on the correlated metal, rather than the metal-insulator transition, and we study the behavior of the Raman response and the inelastic X-ray scattering for a wide variety of electron concentrations and interaction strengths. A number of features arise in the Raman response that are quite interesting. Of course, the results for the nonresonant $B_{1 \mathrm{~g}}$ Raman response are similar to those that have been shown for the optical conductivity ${ }^{11,8}$, but we discuss a number of novel features, including the inelastic scattering of X-rays here. In Section II we present our formalism, results appear in Section III, and we conclude in Section IV.

\section{FORMALISM}

Unlike an optical conductivity experiment, which probes just one symmetry of the two-particle charge excitations, inelastic light scattering can probe a number of different symmetries by placing polarizers on the incident and reflected light. Three common symmetries that are examined are $A_{1 \mathrm{~g}}$ which has the full symmetry of the lattice, $B_{1 \mathrm{~g}}$ which is a d-wave symmetry, and $B_{2 \mathrm{~g}}$ which is another d-wave symmetry (rotated by $45^{\circ}$ ). The Raman response that is related to the optical conductivity by the Shastry-Shraiman relation ${ }^{4,5}$ is the $B_{1 \mathrm{~g}}$ response. Furthermore, the nonresonant Raman scattering in the $B_{2 \mathrm{~g}}$ channel vanishes ${ }^{5}$ (for nearest neighbor only hopping), and in the $A_{1 \mathrm{~g}}$ channel it requires knowledge of the local irreducible charge vertex (which has never been calculated for the Hubbard model), so we provide results only for the $B_{1 \mathrm{~g}}$ sector here.

The Hubbard model ${ }^{9}$ is the simplest model of electronic correlations that possesses a fermi-liquid fixed point for a wide range of parameter space ${ }^{10}$. The fermiliquid forms at low temperature, and often one finds quite anomalous behavior in the transport at higher temperatures, where the fermi-liquid coherence has not yet been established ${ }^{11}$.

The Hubbard Hamiltonian ${ }^{9}$ contains two terms: the electrons can hop between nearest neighbors [with hopping integral $t^{*} /(2 \sqrt{d})$ on a $d$-dimensional hypercubic lattice $\left.^{12}\right]$, and they interact via a screened Coulomb interaction $U$ when they sit on the same site. All energies are measured in units of $t^{*}$. The Hamiltonian is

$$
H=-\frac{t^{*}}{2 \sqrt{d}} \sum_{\langle i, j\rangle, \sigma} c_{i \sigma}^{\dagger} c_{j \sigma}+U \sum_{i} n_{i \uparrow} n_{i \downarrow},
$$


where $c_{i \sigma}^{\dagger}\left(c_{i \sigma}\right)$ is the creation (annihilation) operator for an electron at lattice site $i$ with spin $\sigma$ and $n_{i \sigma}=c_{i \sigma}^{\dagger} c_{i \sigma}$ is the electron number operator. We adjust a chemical potential $\mu$ to fix the average filling of the electrons ( $\mu=$ $U / 2$ at half filling of $\rho_{e}=1.0$ for the electrons).

We treat the model (1) in the infinite-dimensional limit so the electronic properties can be determined by dynamical mean field theory. The starting point is the locality of the single-particle self energy, i.e. the local Green function is the Hilbert transformation of the noninteracting density of states ${ }^{10,8}\left[\rho(\epsilon)=\exp \left(-\epsilon^{2}\right) / \sqrt{\pi}\right.$ on a hypercubic lattice in $d \rightarrow \infty$ ]

$$
G(\omega)=\int_{-\infty}^{\infty} d \epsilon \rho(\epsilon) \frac{1}{\omega+\mu-\Sigma(\omega)-\epsilon}
$$

with $\omega$ approaching the real axis from above.

Formally, $G(\omega)$ can be viewed as the Green's function of an effective single impurity Anderson model. ${ }^{10,8}$ The propagator $G_{0}(\omega)$ for the corresponding effective noninteracting impurity model is then determined by

$$
G_{0}^{-1}(\omega)=G^{-1}(\omega)+\Sigma(\omega) .
$$

Next an Anderson impurity model solver must be employed to determine the local Green's function from $G_{0}(\omega)$ and $U$. Here, we use the numerical renormalization group (NRG) technique ${ }^{13,14}$ to calculate the self energy on the real axis, and then employ Eq. (2) to determine the new Green's function. This algorithm is iterated until the Green's functions converge to a fixed point.

The NRG is based on a logarithmic discretization of the energy axis, i.e. one introduces a parameter $\Lambda>1$ and divides the energy axis into intervals $\pm\left[\Lambda^{-(n+1)}, \Lambda^{-n}\right]$ for $n=0,1, \ldots, \infty^{13,14}$. With some further manipulations one can map the original model onto a semi-infinite chain, which can be solved iteratively by starting from the impurity and successively adding chain sites. Since the coupling between two adjacent sites $n$ and $n+1$ vanishes like $\Lambda^{-n / 2}$ for large $n$, the low-energy states of the chain with $n+1$ sites are determined by a comparatively small number $N_{\text {states }}$ of states close to the ground state of the $n$-site system. In practice, one retains only these $N_{\text {states }}$ from the $n$-site chain to set up the Hilbert space for $n+1$ sites and thus prevents the usual exponential growth of the Hilbert space as $n$ increases. Eventually, after $n_{\mathrm{NRG}}$ sites have been included in the calculation, adding another site will not change the spectrum significantly and one terminates the calculation.

It is obvious, that for any $\Lambda>1$ the NRG constitutes an approximation to the system with a continuum of band states but becomes exact in the limit $\Lambda \rightarrow 1$. Performing this limit is, of course, not possible as one has to simultaneously increase the number of retained states to infinity. One can, however, study the $\Lambda$ - and

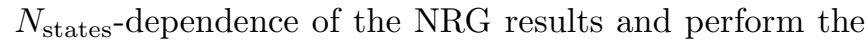
limit $\Lambda \rightarrow 1, N_{\text {states }} \rightarrow \infty$ by extrapolating these data.
Surprisingly one finds that the dependence of the NRG results on $\Lambda$ as well as on the cut-off $N_{\text {states }}$ is extremely mild; in most cases, a choice of $\Lambda=2$ and $N_{\text {states }}=300 \ldots 500$ is sufficient.

While the knowledge of the states is sufficient to calculate thermodynamic properties, the discretization leads to a Green's function consisting of a discrete set of poles and an appropriate coarse-graining procedure has to be applied. ${ }^{15,16}$. Obviously, the NRG is most accurate for energies near the chemical potential, where the logarithmic grid is finest. It is less accurate at energies far away from the chemical potential, because the energy grid is coarser and one uses an asymmetric (logarithmic) broadening for the delta functions. Since our final response functions involve complex convolution integrals containing the interacting Green function, the results are expected to be most accurate in the low-frequency region. At high frequencies one may still expect the qualitative features to be correct, but details like the precise distribution of spectral weight will typically be less reliable. The quantitative errors in both the Green's function and the inelastic light scattering response functions, however, are difficult to estimate.

The nonresonant Raman response in the $\mathrm{B}_{1 \mathrm{~g}}$ channel and the inelastic X-ray scattering along the Brillouinzone diagonal (in the $B_{1 \mathrm{~g}}$ channel) have no vertex corrections ${ }^{19,7,5}$ and are equal to the bare bubbles. The formula for the Raman response has been presented elsewhere ${ }^{5}$ and can also be found from the SS relation ${ }^{4,11,8}$ - the imaginary part of the nonresonant $B_{1 \mathrm{~g}}$ Raman response is

$$
\begin{aligned}
\operatorname{Im} \chi_{B_{1 g}}(\mathbf{q}=0, \nu) & =c \int d \omega[f(\omega)-f(\omega+\nu)] \\
& \times \int d \epsilon \rho(\epsilon) A(\epsilon, \omega) A(\epsilon, \omega+\nu),
\end{aligned}
$$

where $f(\omega)=1 /[1+\exp (\omega / T)]$ is the Fermi function, $A(\epsilon, \omega)=-\operatorname{Im}[1\{\omega+\mu-\Sigma(\omega)-\epsilon\} \pi]$ is the spectral function, and $c$ is a constant.

The inelastic light scattering by a photon with momentum $\mathbf{q}=(q, q, \ldots, q)$ can also be calculated with the bare bubble, because one can show that all vertex corrections vanish here as well ${ }^{20}$. The result depends only on the parameter $X=\cos q$, and is

$$
\begin{aligned}
\chi_{B_{1 g}}(\mathbf{q}, \nu) & =\frac{i}{4 \pi} \int_{-\infty}^{\infty} d \omega\left\{f(\omega) \chi_{0}(\omega ; X, \nu)\right. \\
& -f(\omega+\nu) \chi_{0}^{*}(\omega ; X, \nu)-[f(\omega)-f(\omega+\nu)] \\
& \left.\times \tilde{\chi}_{0}(\omega ; X, \nu)\right\}
\end{aligned}
$$

with

$$
\begin{aligned}
\chi_{0}(\omega ; X, \nu) & =-\int_{-\infty}^{\infty} d \epsilon \rho(\epsilon) \frac{1}{\omega+\mu-\Sigma(\omega)-\epsilon} \frac{1}{\sqrt{1-X^{2}}} \\
& \times F_{\infty}\left(\frac{\omega+\nu+\mu-\Sigma(\omega+\nu)-X \epsilon}{\sqrt{1-X^{2}}}\right),
\end{aligned}
$$


and

$$
\begin{aligned}
\tilde{\chi}_{0}(\omega ; X, \nu) & =-\int_{-\infty}^{\infty} d \epsilon \rho(\epsilon) \frac{1}{\omega+\mu-\Sigma^{*}(\omega)-\epsilon} \frac{1}{\sqrt{1-X^{2}}} \\
& \times F_{\infty}\left(\frac{\omega+\nu+\mu-\Sigma(\omega+\nu)-X \epsilon}{\sqrt{1-X^{2}}}\right) .
\end{aligned}
$$

Here we have $F_{\infty}(z)=\int d \epsilon \rho(\epsilon) /(z-\epsilon)$, which is the Hilbert transform of the noninteracting density of states. Note that the $X=1$ limit of Eq. (5) corresponds to the optical photon $(\mathbf{q}=0)$ and it reduces to the Raman scattering case in Eq. (4) as it must.

\section{RESULTS}

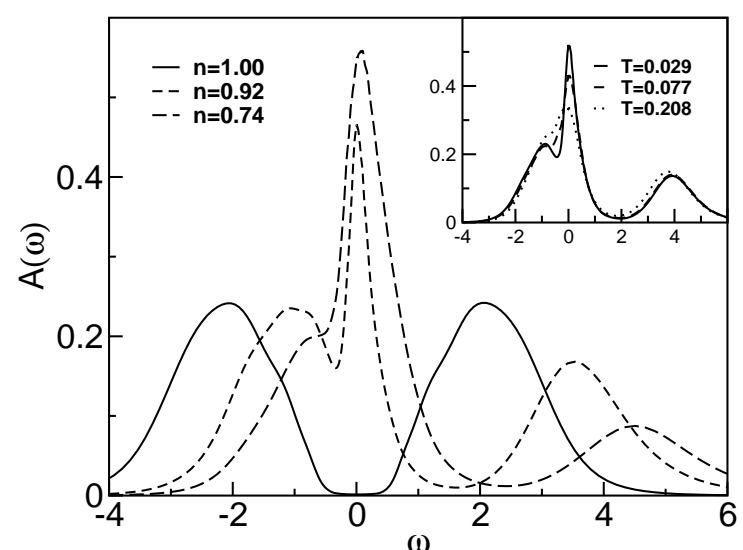

FIG. 1. Evolution of the single-particle DOS for $U=4.24$ with filling for $T=0.029$ (main panel) respectively with temperature for $\rho_{e}=0.92$ (inset). Note especially the strong variation of the quasi-particle peak in both cases. In addition, with decreasing filling there is a strong redistribution of spectral weight from the upper Hubbard band to the Fermi energy. For even lower filling, the upper Hubbard band vanishes completely.

Before discussing the Raman spectra in detail, let us briefly look at the single-particle density of states (DOS). Although this quantity has been extensively discussed in the literature ${ }^{8}$ it is instructive to review the major properties here because they will be needed to interpret the Raman spectra.

The evolution of the DOS for $U=4.24$ upon varying filling and temperature is collected in Fig. 1. Quite generally, one can identify three structures: A comparatively sharp peak at the Fermi energy $\omega=0$, and two broad features, the so-called Hubbard bands, representing incoherent (local) charge excitations (the sharp peak vanishes at $n=1.0$ because the system is in the Mott-insulating state). With decreasing filling (Fig. 1, main panel) these latter structures become less pronounced, the lower one being absorbed into the quasi-particle peak, and the spectral weight of the upper one redistributed to the Fermi level. For still lower filling one would eventually arrive at a single peak at the Fermi level, as in the non-interacting system. For a fixed filling, on the other hand, the variation with temperature (Fig. 1 inset) does not affect the incoherent structures much, but rather leads to a strong variation in the quasi-particle peak at the Fermi energy. Changing the value of $U$ does not lead to qualitatively different features, only the specific values of the filling or temperature, where changes become pronounced, will vary.

We now turn to the discussion of our results for Raman scattering by examining a correlated metal with $U=2.12$. This system is metallic (in the paramagnetic phase) for all electron fillings. The fermi temperature is quite low though, so the quasi-particle peak only appears in the interacting DOS for low enough temperature (c.f. Fig. 1). This has implications for the Raman response as well. We show the Raman scattering at $U=2.12$ for a variety of temperatures in Fig. 2. We plot results for four different electron concentrations (a) $\rho_{e}=1.0$, (b) $\rho_{e}=0.9$, (c) $\rho_{e}=0.8$, and (d) $\rho_{e}=0.6$. Inset in each panel is a blow up of the low-temperature region where the fermi peak appears. The behavior appears qualitively different near half filling and far from half filling. Near half filling, we see a large charge-transfer peak at $\nu \approx 1.5$ present at high temperature, which has a fermiliquid peak separate out of it at low temperature. The fermi peak narrows and is pushed to lower energy as $T$ is lowered, as we expect for a correlated metal as the phase space for particle-hole scattering is reduced. The relative weight of the low energy (fermi-liquid) feature and the charge-transfer feature changes as we move away from half filling. At $\rho_{e}=0.8$, we find a broad highenergy feature, which may be separating into two peaks centered at approximately 1.5 and 2.5 at the lowest temperature. The scattering for $\rho_{e}=0.6$ is even more dramatically modified. We see no large charge transfer peak at high temperature, just a broad low-energy peak, reminiscent of the fermi-liquid peak. As $T$ is lowered, this broad peak narrows and evolves to low energy. As it does so, we see two additional features emerge in the Raman response, corresponding to a mid-infrared bump and a charge transfer bump. Since the interacting DOS typically has a three peak structure (see Fig. 1), with a lower Hubbard band, an upper Hubbard band, and a quasiparticle peak, these three features arise from transitions within the quasiparticle peak, between the quasiparticle peak and the lower band, and between the quasiparticle peak and the upper band. When the electron filling is less than half filled, the quasiparticle peak in the DOS lies well within what would be termed the lower Hubbard band. Finally, we note that the evolution of Raman scattering with doping is an evolution toward weaker correlations as $\rho_{e}$ is reduced, which is precisely what we expect for the Hubbard model, which is most strongly correlated at half filling.

We compare these moderate correlation results to a more strongly correlated system $(U=4.24)$ in Fig. 3. Here three different electron fillings are shown: (a) $\rho_{e}=$ 
1.0; (b) $\rho_{e}=0.9$, and (c) $\rho_{e}=0.8$. At half filling [panel (a)], the system is a correlated insulator, and the Raman response displays the characteristic features of an insulator, including the appearance of low-energy spectral weight at an onset temperature that is smaller than the $T=0$ gap, and an isosbestic point, where the Raman response is independent of temperature (here occurring near $\nu \approx 3$ ). As the system is doped, its behavior changes dramatically, because the doped system is metallic. Here [panels (b) and (c)] we see two peaks at high temperature. As $T$ is lowered, the low-energy peak has a fermi-liquid peak split off and narrow and move to lower energy as $T$ is reduced. The relative weight of the lowenergy features (the fermi-liquid peak and the "mid-IR" peak) to the charge-transfer peak increases as the filling moves away from half filling. We also can see that the fermi-liquid temperature increases as we move away from half filling because of the sharper fermi peak at the same temperature in panel (c) versus panel (b). This is to be expected, because as the relative correlations are reduced, the coherence temperature should increase.

One might be tempted to perform an analysis similar to what was done for the optical conductivity ${ }^{11,8}$, where one examines the relative weights of the fermi peak, the mid-IR bump and the charge transfer peak. Such an analysis is really only feasible for the situation where one has a sum rule satisfied (like the optical conductivity). The multiplication of the optical conductivity by the frequency to produce the Raman response obviously changes the sum rule, and makes such simple identifications more difficult ${ }^{21}$. But the general qualitative feature of more scattering going into the charge transfer peak as the correlations increase certainly still holds. The fermi peak is smaller in $B_{1 \mathrm{~g}}$ Raman scattering, since it is concentrated at small frequencies, and it is reduced by the extra power of frequency that multiplies the optical conductivity to produce the Raman result.

We examine the Raman scattering at constant temperature in a moderately correlated metal $U=2.12$ in Fig. 4. At high temperature [panel (a)], we see a broad charge-transfer-like feature near half filling, but as the electron concentration moves away from 1.0, we see spectral weight shift to low energy and evolve into a sharp fermi liquid peak. For intermediate fillings, we can still see a remnant of the charge-transfer peak at high energy, as a wiggle in the Raman response, but the size of that feature decreases sharply with filling. This picture clearly shows how the fermi temperature depends strongly on electron concentration, as the fermi temperature evolves to become larger than 0.341 around $\rho_{e} \approx 0.6$. At low temperature [panel (b)], we see a strong fermi-liquid peak, that narrows and moves to lower energy with electron filling, as expected. In addition, we see the higher-energy processes are sharpened, with a "mid-IR" feature occurring near $\nu \approx 1$ and a charge-transfer feature at higher energies. Once again, the relative weight of the higherenergy feature gets smaller as we move the filling farther from half filling.
In Fig. 5, we plot the Raman response at fixed $T$ for a strongly correlated system $U=4.24$. In the hightemperature case [panel (a)], we find two prominent peaks, one a charge transfer peak at high energy and one a "mid-IR" peak at lower energy. The temperature is too high to see the fermi-liquid peak forming. We can see, however, the dramatic transfer of relative spectral weight to lower energy as the filling moves farther from half filling, and the charge-transfer peak shrinks. At the lower temperature [panel (b)], we see a sharp charge-transfer peak, and an approximate isosbestic point, where the Raman response is almost independent of electron filling near $\nu \approx U / 2$. Close to the insulating phase, there is very small low-energy spectral weight. As we move away from half filling, the low-energy spectral weight rises, developing into a "mid-IR" feature and having an even lowerenergy fermi peak. This result is similar to what was seen in the optical conductivity ${ }^{11,8}$, where there appeared to be an isosbestic point at about $U / 2$ as a function of doping for fillings close to half filling. The isosbestic point is approximately preserved here, because multiplying by the frequency does not remove such behavior.

We end our results by showing the inelastic light scattering response of $\mathrm{X}$-rays with momenta that run along the Brillouin zone boundary. Fig. 6 shows the moderately correlated metal and Fig. 7 shows the more strongly correlated metal. Let's first start with the moderately correlated results in panel 6 (a). There one sees near the Brillouin zone center that the fermi-liquid peak evolves away from the charge-transfer peak as $T$ is lowered. But once the photon has finite momentum, the fermi-liquid feature is strongly suppressed due to the availability of phase-space to create particle-hole pairs. We see no peak separate out, but the scattering response does appear to sharpen as $T$ is reduced (although it is generically much broader than what is seen at the zone center). In panel (b), which is even more weakly correlated, we find similar behavior except at the zone boundary $(X=-1)$, where the results are counter-intuitive. At higher $T$, we see the low-energy X-ray scattering increase as $T$ decreases, although there is only weak $T$ dependence. But at the lowest temperature, the response is depleted, as if the system is becoming more insulating. This behavior does not seem to occur at other momentum transfers, or in the more strongly correlated case, and we do not have a good understanding for why it happens here, but the scattering of large momentum photons with correlated charge excitations is a complex process that is not easy to infer quantitative results about.

Moving on to the more strongly correlated system in Fig. 7, we see some interesting behavior there as well. Now we can see the remnant of the fermi-liquid peak split-off at low energy surviving out to $X=0.5$, although the peak does not move to lower energy as $T$ is reduced, as it does at the zone center. We see the overall broadening of peaks at finite momentum, and a sharpening of features as $T$ is reduced. The slope of the scattering response at the zone boundary can become quite large at 


\section{CONCLUSIONS}

We have investigated inelastic scattering of light in correlated metals by solving for the dynamical response functions exactly in the infinite-dimensional limit. We examine the Hubbard model and thereby are limited to analyzing nonresonant scattering in the $B_{1 \mathrm{~g}}$ sector and along the Brillouin-zone diagonal. The Raman scattering case corresponds to sitting at the zone center, while the inelastic X-ray scattering allows one to probe momentum transfers along the zone diagonal. Since the $B_{1 \mathrm{~g}}$ response is related to the optical conductivity, many of our results are similar to those found when investigating the optical conductivity, such as the appearance of three generic features corresponding to a fermi-liquid peak, a "midIR" feature, and a charge-transfer peak. The relative weights and temperature dependences of these three features vary with coupling strength and electron filling, but at low enough temperature, we will always see a fermiliquid peak split off and evolve towards zero frequency as $T$ is lowered (except at half filling with sufficiently large $U$ that creates an insulator). When we allow the photon to exchange momentum with the charge excitations, many of the sharp features in the Raman scattering are blurred, and the peaks are broadened. We no longer see the fermi peak evolve at finite momentum transfer, but as the correlations are made stronger, we do sometimes see remnants of the fermi-liquid peak evolution (when $\mathbf{q}$ is close to the zone center). Near the zone boundary, however, there is just a broad, relatively featureless response, whose low-energy slope increases as the system is more correlated and as the temperature is lowered.

To date, there has been limited examinations of electronic Raman scattering or inelastic X-ray scattering of correlated metallic systems. The most studied case is that of the high-temperature superconductors, but they cannot be examined over the full temperature range because they have intervening magnetically ordered, or superconducting phases, which can dramatically change the Raman scattering. Similarly, most resonant inelastic Xray scattering experiments have focused on correlated insulators, because of the difficulty in removing the elastic peak from the response function at the lowest energies, where one might expect interesting fermi-liquid features to appear. Our theoretical results make a number of predictions for how the temperature, doping, and correlation strength dependence of inelastic light scattering varies in correlated metals and we believe it might be possible to see some of these features in heavy-fermion materials, which have not yet been exhaustively studied with these techniques.
J.K.F. acknowledges support of the National Science Foundation under grants DMR-9973225 and DMR0210717. T.P.D. acknowledges support from the National Research and Engineering Council of Canada and PREA. R.B. and Th.P. acknowledge support by the Deutsche Forschungsgemeinschaft, through the Sonderforschungsbereich 484 . We also acknowledge useful discussions with S.L. Cooper, R. Hackl, J.P. Hill, M.V. Klein, and S. Shastry.

${ }^{1}$ T. Katsufuji, Y. Tokura, T. Ido, and S. Uchida, Phys. Rev. B 48, 16131 (1993); X. K. Chen, J.G. Naeini, K.C. Hewitt, J.C. Irwin, R. Liang, W.N. Hardy, Phys. Rev. B 56, R513 (1997); J.G. Naeini, X.K. Chen, J.C. Irwin, M. Okuya, T. Kimura, and K. Kishio, Phys. Rev. B 59, 9642 (1999).

${ }^{2}$ M. Opel, R. Nemetschek, C. Hoffmann, R. Philipp, P.F. Müller, R. Hackl, I. Tütto, A. Erb, B. Revaz, E. Walker, H. Berger, and L. Forró, Phys. Rev. B 61, 9752 (2000); F. Venturini, M. Opel, T. P. Devereaux, J. K. Freericks, I. Tüttö, B. Revaz, E. Walker, H. Berger, L. Forró and R. Hackl, Phys. Rev. Lett. 89, 107003 (2002).

${ }^{3}$ M. Rübhausen, O.A. Hammerstein, A. Bock, U. Merkt, C.T. Rieck, P. Guptasarma, D.G. Hinks, M.V. Klein, Phys. Rev. Lett. 82, 5349 (1999); S. Sugai and T. Hosokawa, Phys. Rev. Lett. 85, 1112 (2000).

${ }^{4}$ B. S. Shastry and B. I. Shraiman, Phys. Rev. Lett. 65, 1068 (1990); Int. J. Mod. Phys. B 5, 365 (1991).

${ }^{5}$ J.K. Freericks and T.P. Devereaux, Phys. Rev. B 64, 125110 (2001).

${ }^{6}$ J.K. Freericks, T.P. Devereaux, and R. Bulla, Acta Phys. Pol. B 32, 3219 (2001); Phys. Rev. B 64, 233114 (2001).

${ }^{7}$ J.K. Freericks and T.P. Devereaux, J. Cond. Phys. (Ukraine) 4, 149 (2001).

8 Th. Pruschke, M. Jarrell, and J. K. Freericks, Adv. Phys. 44, 187 (1995).

${ }^{9}$ J.C. Hubbard, Proc. Royal Soc. London, Ser. A 276, 238 (1963).

10 A. Georges, G. Kotliar, W. Krauth, and M. J. Rozenberg, Rev. Mod. Phys. 68, 13 (1996).

11 Th. Pruschke, D.L. Cox, and M. Jarrell, Phys. Rev. B 47, 3553 (1993); Europhys. Lett. 21, 593 (1993); M. Jarrell and Th. Pruschke, Phys. Rev. B 49, 1458 (1994).

${ }^{12}$ W. Metzner and D. Vollhardt, Phys. Rev. Lett. 62, 324 (1989).

13 A. C. Hewson, The Kondo Problem to Heavy Fermions, Cambridge Univ. Press (1993).

${ }^{14}$ K.G. Wilson, Rev. Mod. Phys. 47, 773 (1975); H.R. Krishna-murthy, J.W. Wilkins and K.G. Wilson, Phys. Rev. B21, 1003 (1980); 21, 1044 (1980).

${ }^{15}$ O. Sakai, Y. Shimizu and T. Kasuya, J. Phys. Soc. Jpn. 58, 3666 (1989); T.A. Costi, A. Hewson and V. Zlatić, J. Phys.: Cond. Matter 6, 2519 (1994). 
${ }^{16}$ R. Bulla, Th. Pruschke and A.C. Hewson J. Phys.: Cond. Matter 910463 (1997); J. Phys.: Cond. Matter 10, 8365 (1998); R. Bulla, Phys. Rev. Lett. 83, 136 (1999); R. Bulla, T. A. Costi, and D. Vollhardt, Phys. Rev. B 64, 045103 (2001).

17 J. M. Luttinger, Phys. Rev. 119, 1153 (1960).

${ }^{18}$ E. Müller-Hartmann, Int. J. Phys. B 3, 2169 (1989).

19 A. Khurana, Phys. Rev. Lett., 64, 1990 (1990).

20 There is a special problem in calculating the inelastic Xray scattering at the zone diagonal point corresponding to $X=-1$. This occurs because there is a pole in the integrand that lies arbitrarily close to the real axis. Hence caution must be used in numerically evaluating the integrand. The effect of this pole is not too dramatic on the analytic continuation aside from introducing some extraneous high-frequency oscillations in the imaginary part of the scattering response function. The oscillations are typically small in amplitude and can be corrected by properly handling the pole term in the numerical integrations.

${ }^{21}$ J. Kosztin and A. Zawadowski, Sol. State Commun. 78, 1029 (1991).

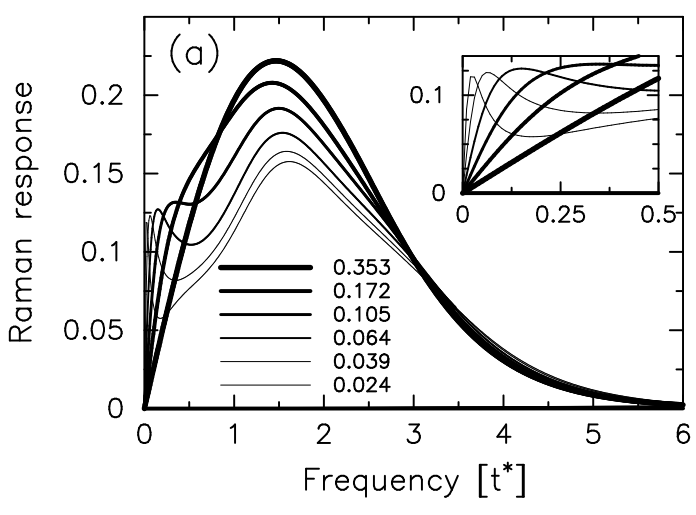

Freericks, et al, Phys. Rev. B, Fig. 1(a)

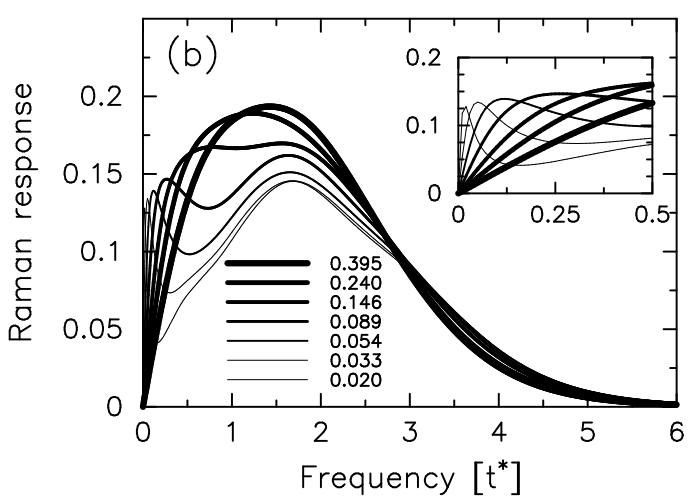

Freericks, et al, Phys. Rev. B, Fig. 1(b)

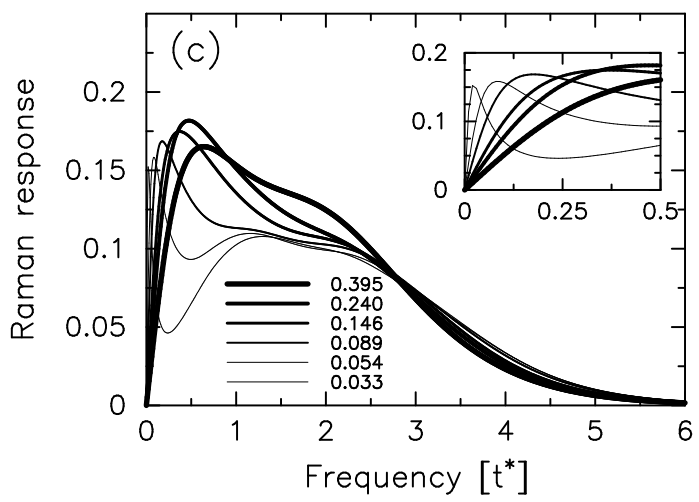

Freericks, et al, Phys. Rev. B, Fig. 1(c)

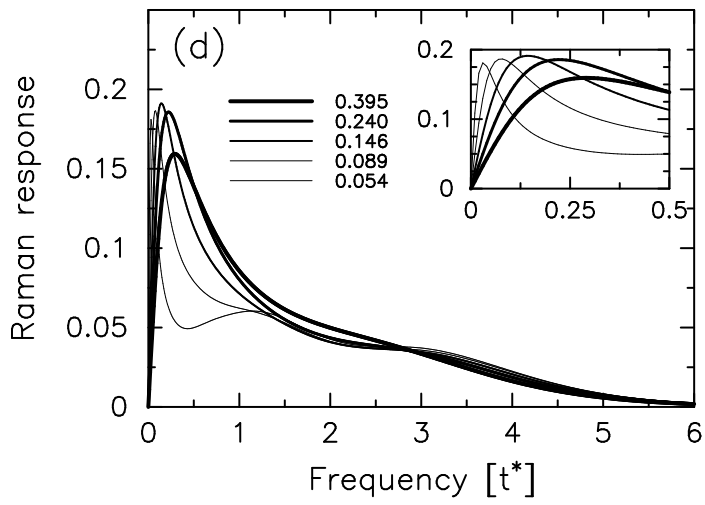

Freericks, et al, Phys. Rev. B, Fig. 1(d)

FIG. 2. Raman response for different temperatures at $U=2.12$. The different panels refer to different electron fillings: (a) $\rho_{e}=1.0$; (b) $\rho_{e}=0.9$; (c) $\rho_{e}=0.8$; and (d) $\rho_{e}=0.6$. The numbers in the legends label the temperature.

6 Inset into each panel is a blow-up of the low-frequency region to show the fermi-liquid peak formation and evolution with $T$. 


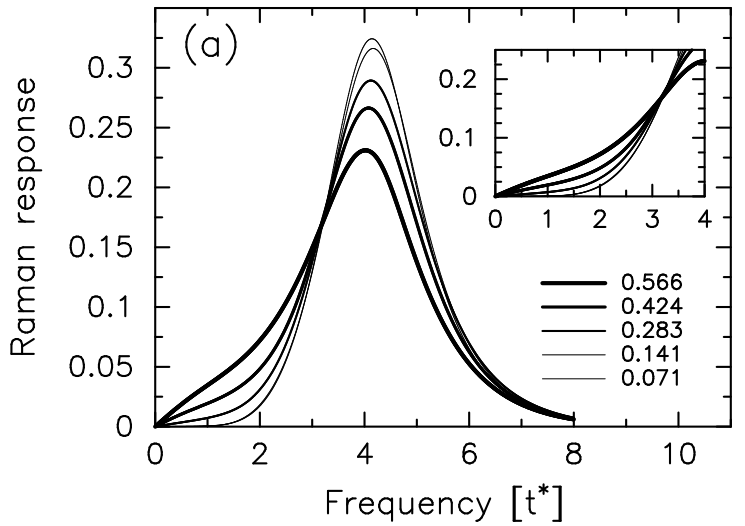

Freericks, et al, Phys. Rev. B, Fig. 2(a)

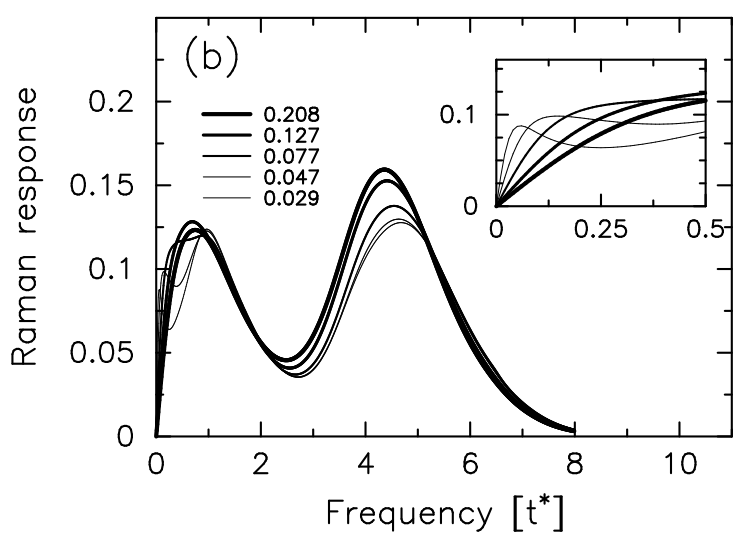

Freericks, et al, Phys. Rev. B, Fig. 2(b)

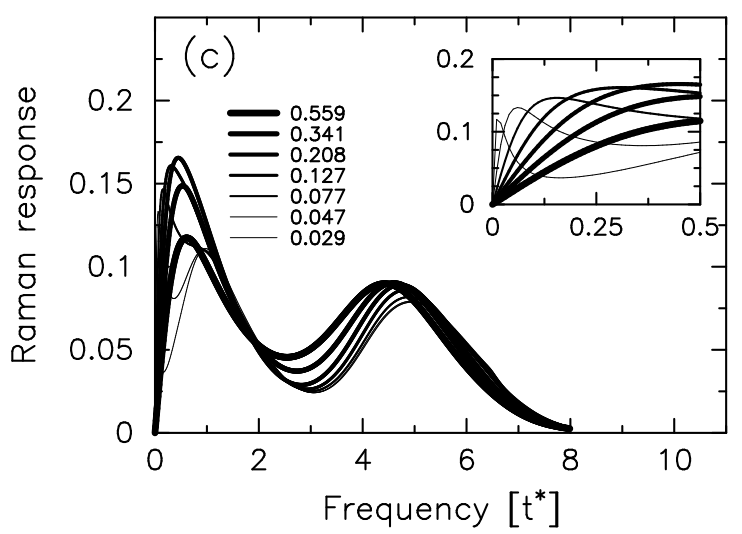

Freericks, et al, Phys. Rev. B, Fig. 2(c)

FIG. 3. Raman response for different temperatures at $U=4.24$. The different panels refer to different electron fillings: (a) $\rho_{e}=1.0$; (b) $\rho_{e}=0.9$; and (c) $\rho_{e}=0.8$. The numbers in the legends label the temperature. Inset into each panel is a blow-up of the low-frequency region to show the fermi-liquid peak formation and evolution with $T$.

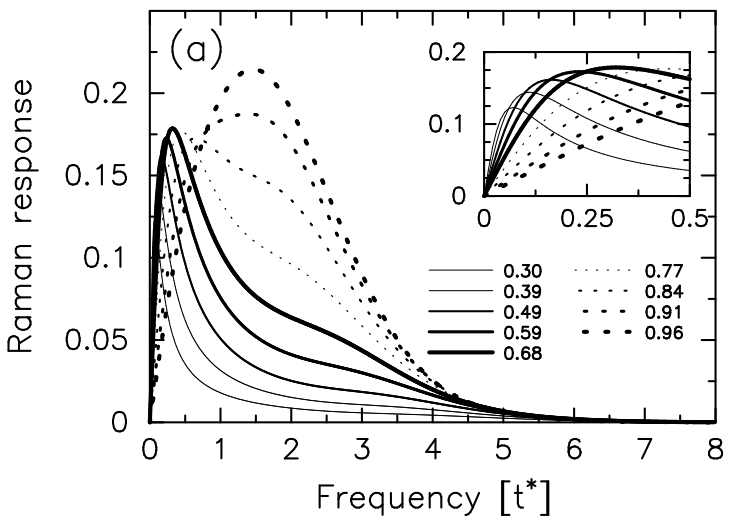

Freericks, et al, Phys. Rev. B, Fig. 3(a)

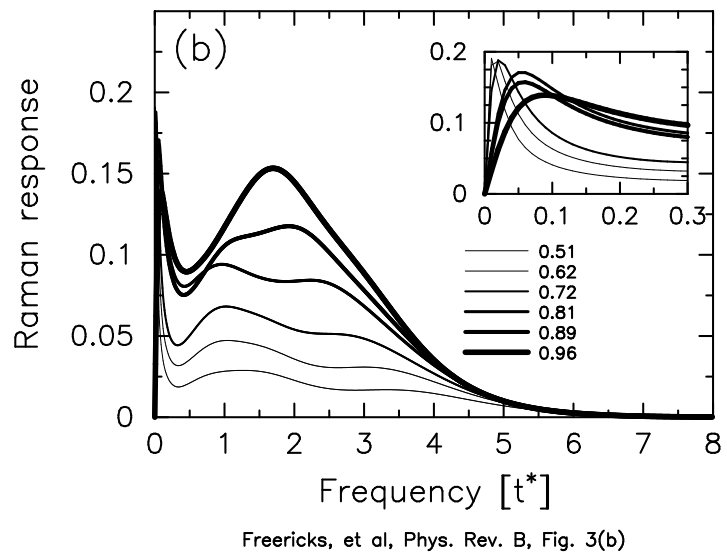

FIG. 4. Raman response for different fillings at $U=2.12$ and constant temperature. The different panels refer to different $T$, (a) $T=0.341$ and (b) $T=0.0472$. The numbers in the legends label the electron filling. Inset into each panel is a blow-up of the low-frequency region to show the fermi-liquid peak formation and evolution with electron filling. 

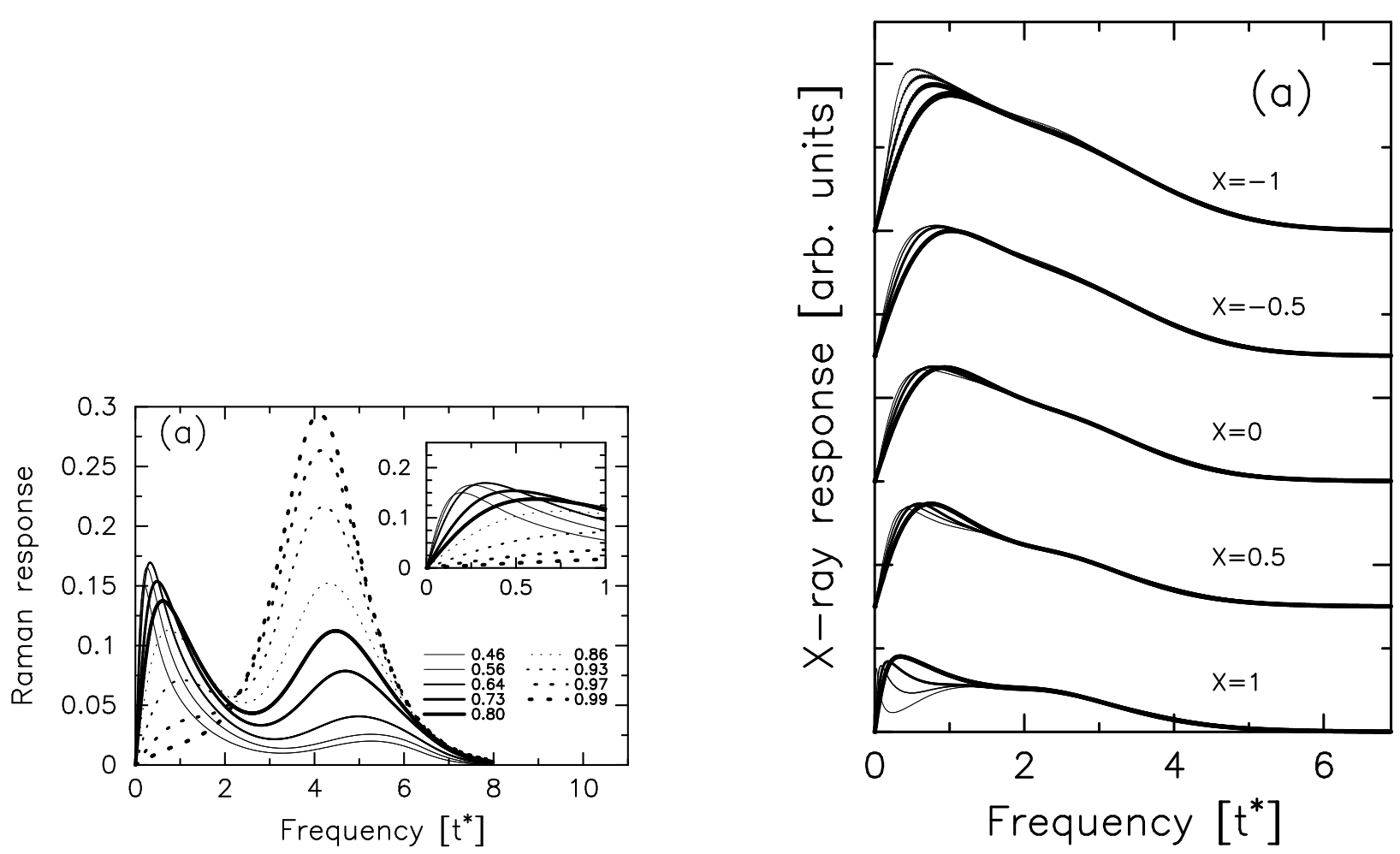

Freericks, et al, Phys. Rev. B, Fig. 4(a)

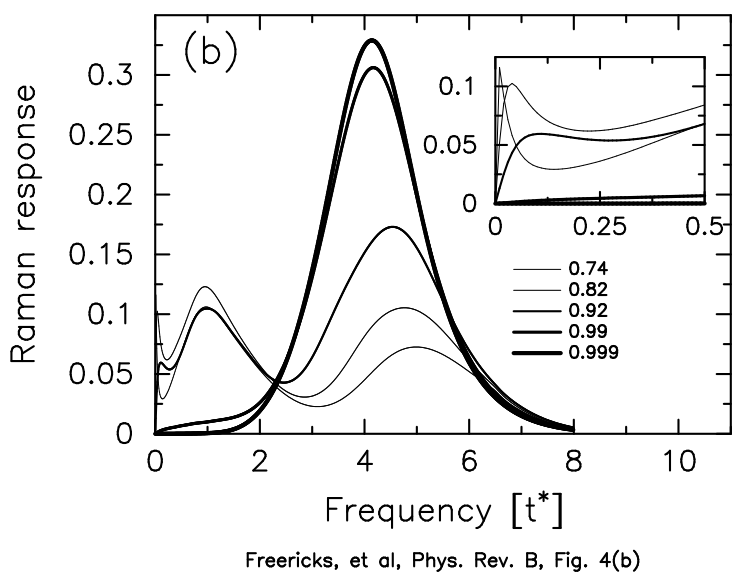

FIG. 5. Raman response for different fillings at $U=4.24$ and constant temperature. The different panels refer to different $T$, (a) $T=0.341$ and (b) $T=0.0248$. The numbers in the legends label the electron filling. Inset into each panel is a blow-up of the low-frequency region to show the fermi-liquid peak formation and evolution with electron filling.

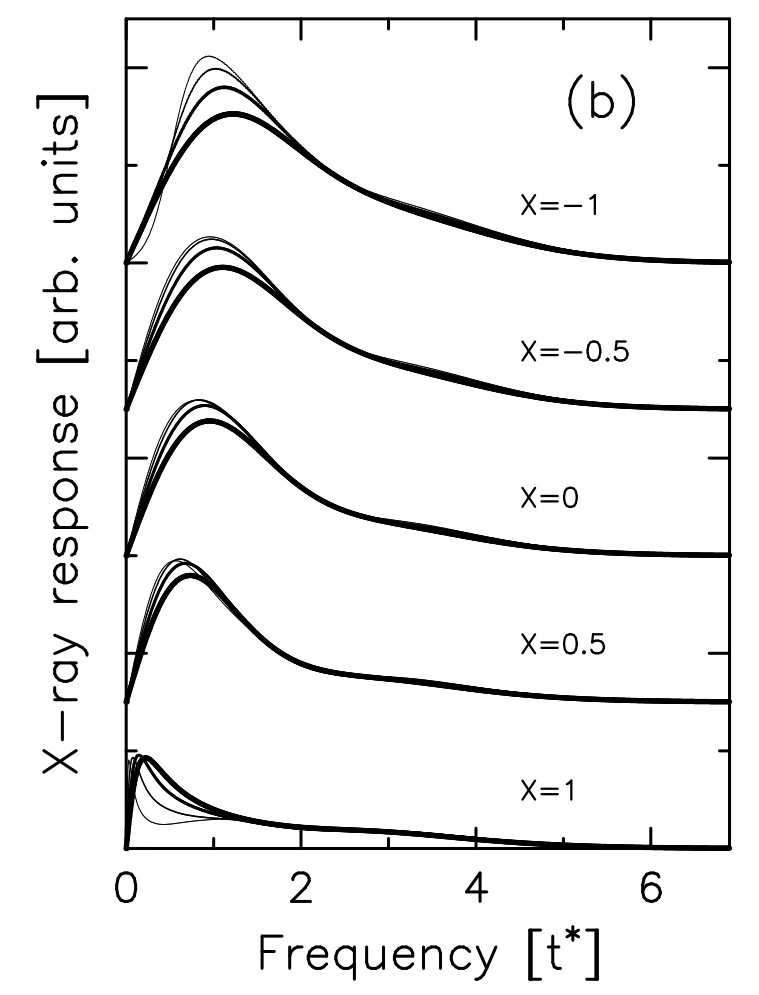

FIG. 6. Inelastic light scattering at $U=2.12$ and (a) $\rho_{e}=0.8$ and (b) $\rho_{e}=0.6$ for different temperatures. Five values of the transferred photon momentum are plotted, each shifted by an appropriate amount, and running from the zone center to the zone boundary along the zone diagonal. The results are for the $B_{1 \mathrm{~g}}$ sector only. In panel (a) the temperature decreases as the thickness decreases and ranges from 0.146 to 0.089 to 0.054 to 0.033 . In panel (b) the temperature also decreases as the thickness decreases and ranges from 0.240 to 0.146 to 0.089 to 0.054 . 


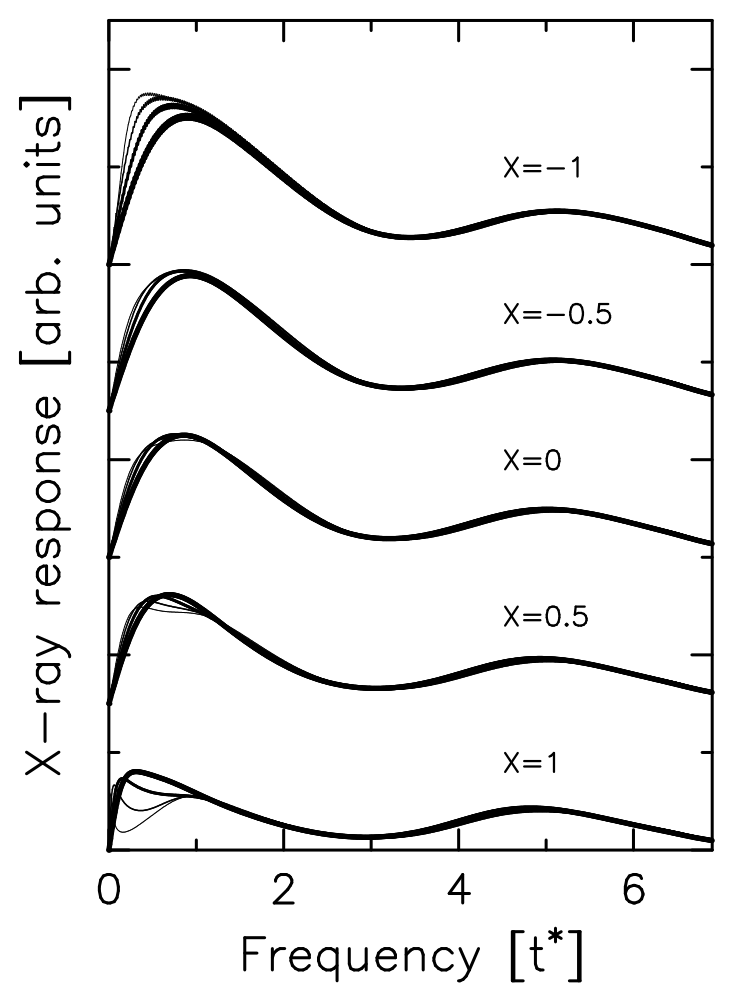

FIG. 7. Inelastic light scattering at $U=4.24$ and $\rho_{e}=0.8$ for different temperatures. Five values of the transferred photon momentum are plotted, each shifted by an appropriate amount, and running from the zone center to the zone boundary along the zone diagonal. The results are for the $B_{1 \mathrm{~g}}$ sector only. The temperature decreases with decreasing thickness and ranges from 0.127 to 0.077 to 0.047 to 0.029 . 